\title{
The Influence of Smoking, Gender, and Family History on Colorectal Adenomas
}

\author{
Tracy Onega, ${ }^{1,2}$ Martha Goodrich, ${ }^{1,2}$ Allen Dietrich,, ${ }^{1,2}$ and Lynn Butterly ${ }^{2,3}$ \\ ${ }^{1}$ Department of Community and Family Medicine, Dartmouth Medical School, Hanover, Lebanon, NH 03756, USA \\ ${ }^{2}$ Norris Cotton Cancer Center, Dartmouth Hitchcock Medical Center, Lebanon, NH 03756, USA \\ ${ }^{3}$ Section of Gastroenterology, Department of Medicine, Dartmouth Medical School, Hanover, Lebanon, NH 03756, USA \\ Correspondence should be addressed to Tracy Onega, onega@dartmouth.edu
}

Received 19 January 2010; Revised 26 March 2010; Accepted 22 April 2010

Academic Editor: Hans-Olov Adami

Copyright (๑) 2010 Tracy Onega et al. This is an open access article distributed under the Creative Commons Attribution License, which permits unrestricted use, distribution, and reproduction in any medium, provided the original work is properly cited.

Evidence independently links smoking, family history, and gender with increased risk of adenomatous polyps. Using data from the New Hampshire Colonoscopy Registry (2004-2006), we examined the relation of combined risk factors with adenoma occurrence in 5,395 individuals undergoing screening colonoscopy. Self-reported data on smoking, family history and other factors were linked to pathology reports identifying adenomatous polyps and modeled with multiple logistic regression. In adjusted models a $>15$ pack-year smoking history increased the likelihood of an adenoma (OR $=1.54,95 \%$ CI $1.28-1.86$ ), although $\leq 15$ packyears did not $(\mathrm{OR}=1.07,95 \% \mathrm{CI} 0.87-1.32)$. Gender-stratified models showed a significantly increased risk of adenoma at lower smoking exposure even for men $(\mathrm{OR}=1.32 ; 95 \% \mathrm{CI}: 1.00-1.76)$, but not for women (OR = 0.85; 95\% CI:0.61-1.14). An ordered logistic regression model of adenoma occurrence showed a smoking history of $\geq 15$ pack-years associated with $61 \%$ higher odds of adenoma at successively larger size categories (95\% CI 1.34-1.93). For individuals with a family history of colorectal cancer, smoking does not further increase the risk of adenomas. Smoking duration is linked to occurrence and size of adenoma, especially for men.

\section{Introduction}

Identification of risk factors for colonic adenomas is important in order to risk stratify individuals for colorectal neoplasia and to guide the most appropriate screening recommendations. Many studies have linked age, gender, smoking, family history, and other factors, with risk of adenoma occurrence [1-7]. Less well studied are potential modifying interactions among risk factors. Examining the influence of concurrent risk factors on adenomas is important to clinical practice, since patients often present with multiple risk factors, which physicians must consider to optimize individual screening practices. The roles of family history, smoking, and gender in adenoma development have received much attention, with significant associations demonstrated for each $[5,7,8]$. Smoking has inconsistently been shown to be an important risk factor for adenoma occurrence $[3,5,6,8-10]$ although a recent meta-analysis showed a significant effect [9]. Family history of colorectal cancer or polyps is known to be associated with a higher likelihood of developing adenomas, $[5,8]$ as is male sex $[8,10]$. How these factors, in combination, relate to adenoma risk is not well characterized. Associations of risk factor combinations more closely capture clinical presentation of individuals and should be considered to identify patients at the greatest risk for neoplastic colonic lesions.

The aim of this study was to test two hypotheses related to risk of adenomatous polyp occurrence: (1) smoking is associated with greater incremental risk among individuals with a relevant family history than those without; (2) smoking results in greater increase in risk of adenomas among men than women. We conducted a cross-sectional study to examine the combined influences of smoking, gender, and family history on adenomatous polyp risk in patients undergoing screening colonoscopy. We characterized individuals according to smoking status, gender, and family history of colorectal cancer or polyps and analyzed them for significant differences in personal polyp occurrence. 
We also developed logistic regression models to estimate the likelihood of adenomatous polyps as a function of smoking, gender, and family history. Our study is important in its ability to examine the interaction of several important risk factors which will further inform risk assessment of patients.

\section{Materials and Methods}

2.1. Study Subjects and Data Collection. We used data from the design phase (2004-2006) of the New Hampshire Colonoscopy Registry (NHCR), including completed colonoscopies for 7611 individuals, and pathology reports on 1739 of them. The NHCR is described in detail elsewhere [11]. Personal data were collected using a patient questionnaire administered prior to the exam, which ascertained demographic characteristics (age, race, educational level achieved, marital status, and type of health care coverage), risk factors (personal and/or family history of colorectal cancer, polyps, including familial adenomatous polyposis, hereditary nonpolyposis colon cancer, or other familial polyposis, and height and weight), and other health history (use of aspirin, vitamins and calcium, smoking history, alcohol use, and exercise routine). A second data collection form was completed by endoscopists after the colonoscopy and recorded the indication for the procedure being performed (screening for no history, or symptoms, screening for family history, surveillance for patient-related history or diagnostic exam for symptoms) and all other procedure-related information including number and location of polyps removed. Pathology reports for polyps removed during colonoscopy examinations were matched to consenting patients. All data collection and utilization procedures were approved by the Committee for Protection of Human Subjects at Dartmouth Medical School.

We included only colonoscopy procedures which were recorded by the endoscopist as having a screening indication (including surveillance). We did not include exams with a diagnostic indication, including evaluation of symptoms. Exclusion criteria included persons reporting a history of familial adenomatous polyposis $(N=39)$, hereditary nonpolyposis colon cancer $(N=46)$, ulcerative colitis or Crohn's disease $(N=118)$, or a personal history of polyps or colorectal cancer $(N=1160)$. Thus, colonoscopies performed for surveillance rather than screening were excluded. Also excluded were individuals for whom data collection was not complete during the study period ( $\operatorname{sex} N=116$, age $N=317$, smoking history $N=50$, pathology $N=370$ ).

All patient data, including pathology reports, were linked by patient identification number. Multiple colonoscopies for individuals were summarized at the patient level using an algorithm to capture all response variables of interest over time. Only 72 of the 7683 colonoscopies performed were for the same individual. In the case of nonconcordant responses over time, such as change in smoking status, dates of procedure were linked to pathology reports, and decision rules were applied. The most frequent response was used, or the first chronologically if frequencies of a given response were equal.
TABLE 1: Characteristics of the study population undergoing screening colonoscopy in the New Hampshire Colonoscopy Registry from 2004-2006.

\begin{tabular}{lc}
\hline Total & $\mathrm{N}(\%) 5,395$ \\
\hline Smoking & $2,865(53)$ \\
No pack-years & $1,243(23)$ \\
15 pack-years or fewer & $1,287(24)$ \\
More than 15 pack-years & \\
Family History & \\
$\quad$ Negative & $4,649(86)$ \\
Positive & $746(14)$ \\
Gender & \\
Male & $2,284(42)$ \\
Female & $3,111(58)$ \\
Adenoma & \\
None & $4,689(87)$ \\
One or more & $706(13)$ \\
Adenoma Size & \\
No Adenoma & $4,672(87)$ \\
$\leq 5$ mm & $318(6)$ \\
$5-9$ mm & $268(5)$ \\
$\geq 1 \mathrm{~cm}$ & $120(2)$ \\
& Mean (SO) \\
Mean Age (yrs.) & $56( \pm 11)$ \\
\hline
\end{tabular}

${ }^{a}$ Family history was defined as a reported diagnosis of colon or rectal cancer in a mother, father, sibling, or child of the patient.

TABLE 2: Self-reported smoking history among men and women with colonoscopy in the New Hampshire Colonoscopy Registry from 2004-2006.

\begin{tabular}{lccc}
\hline & \multicolumn{3}{c}{ Smoking History } \\
& No Pack-Years & $\leq 15$ Pack-Years & $>15$ Pack-Years \\
\hline Men & $1135(50)$ & $492(21)$ & $658(29)$ \\
Women & $1730(56)$ & $751(24)$ & $630(20)$ \\
\hline
\end{tabular}

2.2. Key Variable Definition. Smoking exposure was defined by pack-years: none, 15 or fewer, more than 15. Family history was defined as a reported diagnosis of colon or rectal cancer in one or more first degree relatives (mother, father, sibling, or child of the patient). Polyps classified as adenomas included tubular adenoma, tubular-villous adenoma, villous adenoma, or mixed polyps. Concurrent adenomatous and hyperplastic polyps were identified by having polyp specimens with each histological classification during the study period. Pathology reports indicating a mixed polyp were classified as a single polyp at the highest grade reported. We included health history and behavioral variables which are thought to be risk factors or risk modifiers for polyp occurrence, specifically: aspirin use [7], alcohol consumption $[5,7,10]$, exercise $[5,7]$, vitamin use [5], supplemental calcium $[5,10]$, and family history of colorectal cancer or polyps $[7,8]$. To maintain robust analyses in regard to sample size, we classified use of aspirin, vitamins, calcium supplementation, and exercise on a binary 
TABle 3

(a) Comparison of adenoma occurrence among men and women in a colorectal screening population in relation to smoking history.

\begin{tabular}{|c|c|c|c|c|c|c|c|c|}
\hline \multicolumn{6}{|c|}{ Men $(\mathrm{N}$, column $\%)$} & \multicolumn{3}{|c|}{ Women $(\mathrm{N}$, column \%) } \\
\hline Adenoma Status & No Pack-Years & $\leq 15$ Pack-Years & $>15$ Pack-Years & $P^{\mathrm{a}}$ & No Pack-Years & $\leq 15$ Pack-Years & $>15$ Pack-Years & $P^{\mathrm{a}}$ \\
\hline None & $969(85)$ & $402(82)$ & $514(78)$ & $<001$ & $1,570(91)$ & $693(92)$ & $541(86)$ & 06 \\
\hline One or more & $166(15)$ & $90(18)$ & $143(22)$ & & $160(9)$ & $58(8)$ & $89(14)$ & \\
\hline
\end{tabular}

(b) Comparison of adenomas among a colorectal screening population with and without a family history of colorectal cancer or polyps, in relation to smoking status.

\begin{tabular}{|c|c|c|c|c|c|c|c|c|}
\hline \multirow[b]{2}{*}{ Adenoma Status } & \multicolumn{3}{|c|}{ No Family History } & \multicolumn{5}{|c|}{ Family History } \\
\hline & No Pack-Years & $\leq 15$ Pack-Years & $>15$ Pack-Years & $P^{\mathrm{a}}$ & No Pack-Years & $\leq 15$ Pack-Years & $>15$ Pack-Years & $P^{\mathrm{a}}$ \\
\hline None & $2,195(90)$ & $935(88)$ & $944(83)$ & \multirow{2}{*}{$<.001$} & $344(83)$ & $160(86)$ & $111(75)$ & \multirow{2}{*}{.019} \\
\hline One or more & $257(10)$ & $123(12)$ & $195(17)$ & & $69(17)$ & $25(14)$ & $37(25)$ & \\
\hline
\end{tabular}

${ }^{\text {a }} P$-values based on Pearson's $\chi^{2}$.

scale (either $<$ or $\geq$ one time/week). Alcohol consumption was categorized as follows: none, 1-8 drinks/week, $\geq 9$ drinks/week.

2.3. Analysis. Smokers and nonsmokers were stratified by gender and family history separately, and evaluated for significant differences in adenoma occurrence with Pearson's $\chi^{2}$. Univariate logistic regressions were performed for each exposure and likelihood of adenoma. We developed a multivariate logistic model of adenoma occurrence, adjusting for important covariates. We stratified by gender and by family history and performed multivariate logistic regression to evaluate the significance of smoking for these stratified groups in relation to risk of adenoma. We also formally tested for interaction of family history with smoking and of sex with smoking by creating an interaction term based on a dichotomous smoking response (ever versus never) and testing in the models using a likelihood ratio test. Ordered logistic regression models were used to test the effect of smoking on size of adenoma, with size categories: no adenoma, $<5 \mathrm{~mm}, 5-9 \mathrm{~mm}$, and $\geq 1 \mathrm{~cm}$, as the ordered dependent outcomes. Smoking was evaluated as both a dichotomous (ever/never) and categorical (pack years: none, $\leq 15,>15$ ) predictor variable in ordered logistic regression models. We tested the proportionality of odds assumption using a likelihood ratio test to confirm the validity of the ordinal logistic regression model. The models were adjusted for significant covariates. Interaction terms were not included in the ordinal logistic models because of the threecategory smoking exposure. All analyses were performed using Stata 10.0 for Windows (Stata Corp., College Station, TX) statistical software.

\section{Results}

A total of 5,395 individuals undergoing screening colonoscopy were included in the analyses. Characteristics of the study population based on smoking status are presented in Table 1. Nonsmokers comprised just over half of the study population. The smokers were equally represented by those with 15 or fewer pack-years and those with more than 15 pack-years, although men had greater smoking history than women (Table 2). Approximately $14 \%$ of study subjects reported a family history of colorectal cancer. More women than men were in the study population ( $58 \%$ versus $42 \%)$ (Table 1).

Differences in adenoma occurrence between men and women with regard to smoking status showed similar patterns (Table 3(a)). For men and women, smokers with greater than a 15 pack-year history had a significantly higher proportion of adenomas compared to nonsmokers. Regardless of smoking status, men had a higher proportion of adenomas than women.

We also examined the influence of smoking in those individuals with and without a family history (Table 3(b)). When considering patients with no family history, smokers with $>15$ pack-years had a significantly greater proportion of adenomas compared to nonsmokers (smokers: $17 \%$ versus nonsmokers: $10 \%, P<.001)$. The same additive effect was seen among patients with a family history, although with higher proportions of adenoma occurrence (25\% versus $17 \%$, Table 3(b)).

To examine how the key risk factors, gender and family history might modify the influence of smoking on adenoma occurrence, we developed stratified logistic regression models (Table 4). Stratifying by gender and adjusting for age and family history, we found that a 15- or fewer packyear smoking history was associated with a $32 \%$ increased likelihood of adenoma compared with nonsmokers among men $(\mathrm{OR}=1.32$; 95\% CI 1.00-1.76). The odds of adenoma among male smokers with more than 15 pack-years of exposure were $55 \%$ higher than nonsmokers $(\mathrm{OR}=1.55$; 95\% CI 1.21-2.00). There was no significant increase in adenomas among female smokers in the lowest pack-year group compared to nonsmokers $(\mathrm{OR}=1.85$; $95 \% \mathrm{CI} 0.61-$ 1.14). Women with more than 15 pack-years of smoking had a $55 \%$ increased odds of adenoma compared to nonsmokers $(\mathrm{OR}=1.55 ; 95 \% \mathrm{CI} 1.17-2.05)$. The likelihood ratio test for interaction of gender with a dichotomous smoking response was not significant. 
TABle 4: Stratified logistic regression models of smoking and adenoma occurrence among individuals undergoing screening colonoscopy in the New Hampshire colonscopy Registry from 2004-2006.

\begin{tabular}{|c|c|}
\hline Model & $\begin{array}{l}\text { Adenoma v. No Adenoma } \\
\text { Odds Ratio }(95 \% \mathrm{Cl})^{\mathrm{a}}\end{array}$ \\
\hline \multicolumn{2}{|l|}{ Full Model $(n=5,395)$} \\
\hline \multicolumn{2}{|l|}{ Smoking } \\
\hline No pack-years & 1.00 (referent) \\
\hline 15 pack-years or fewer & $1.07(0.87-1.32)$ \\
\hline More than 15 pack-years & $1.54(1.28-1.86)^{\ddagger}$ \\
\hline \multicolumn{2}{|l|}{ Sex } \\
\hline Men & 1.00 (referent) \\
\hline Women & $0.53(0.45-0.62)^{\ddagger}$ \\
\hline \multicolumn{2}{|l|}{ Family History ${ }^{\mathrm{b}}$} \\
\hline Negative & 1.00 (referent) \\
\hline Positive & $1.73(1.40-2.15)^{\ddagger}$ \\
\hline \multicolumn{2}{|l|}{ Stratified by Sex } \\
\hline \multicolumn{2}{|l|}{ Male $(n=2,284)$} \\
\hline No pack-years & 1.00 (referent) \\
\hline 15 pack-years or fewer & $1.32(1.00-1.76)^{\dagger}$ \\
\hline More than 15 pack-years & $1.55(1.21-2.00)^{\ddagger}$ \\
\hline \multicolumn{2}{|l|}{ Female $(n=3,111)$} \\
\hline No pack-years & 1.00 (referent) \\
\hline 15 pack-years or fewer & $0.85(0.61-1.14)$ \\
\hline More than 15 pack-years & $1.55(1.17-2.05)^{\ddagger}$ \\
\hline \multicolumn{2}{|l|}{ Stratified by Family History } \\
\hline \multicolumn{2}{|l|}{ Negative $(n=4,649)$} \\
\hline No pack-years & 1.00 (referent) \\
\hline 15 pack-years or fewer & $1.16(0.92-1.46)$ \\
\hline More than 15 pack-years & $1.56\left(1.27-1.91^{\ddagger}\right)$ \\
\hline \multicolumn{2}{|l|}{ Positive $(n=746)$} \\
\hline No pack-years & 1.00 (referent) \\
\hline 15 pack-years or fewer & $0.77(0.47-1.26)$ \\
\hline More than 15 pack-years & $1.50(0.94-2.38)$ \\
\hline
\end{tabular}

a Odds ratios are adjusted for age in all models, and for family history in the sex-stratified models, and for sex in the family history-stratified models.

${ }^{b}$ Family history was defined as a reported diagnosis of colon or rectal cancer in a mother, father, sibling, or child of the patient.

${ }^{\dagger} P=.05$.

${ }^{\ddagger} P \leq .001$.

We compared the effect of smoking among individuals with and without a family history, while adjusting for age and gender. Among patients with no family history, a 15or fewer pack-year smoking history did not significantly increase the risk of adenoma. More than 15 pack-years of smoking increased the odds of adenoma occurrence by $56 \%$ $(\mathrm{OR}=1.56 ; 95 \%$ CI $1.27-1.91)$. For patients with a family history, smoking did not significantly add to the risk of adenoma, although the higher pack-year group showed a nonsignificant trend towards increased risk $(\mathrm{OR}=1.50$; 95\% CI 0.94-2.38). Our formal test of interaction between smoking and family history did not show significance for the interaction term in the adjusted logistic regression models.
TABLE 5: An ordered logistic regression model of smoking and adenoma occurrence among individuals $(n=5,395)$ undergoing screening colonoscopy in the New Hampshire Colonscopy Registry from 2004-2006.

\begin{tabular}{lc}
\hline Variable in full model ${ }^{\mathrm{c}}$ & $\begin{array}{c}\text { Increasing Size Category }{ }^{\mathrm{a}} \text { of } \\
\text { Adenoma v. No Adenoma } \\
\text { Odds Ratio }(95 \% \mathrm{CI})\end{array}$ \\
\hline Smoking & 1.00 (referent) \\
$\quad$ No pack-years & $1.10(0.89-1.34)$ \\
15 pack-years or fewer & $1.61(1.34-1.93)^{*}$ \\
$\quad$ More than 15 pack-years & 1.00 (referent) \\
Sex & $0.54(0.46-0.63)^{*}$ \\
Male & $1.02(1.01-1.03)^{*}$ \\
Female & 1.00 (referent) \\
Age (years) & $1.69(1.37-2.08)^{*}$ \\
Family history & \\
$\quad$ Negative & Positive
\end{tabular}

a Size categories include $<5 \mathrm{~mm}, 5-9 \mathrm{~mm}, \geq 1 \mathrm{~cm}$; odds ratios represent the proportional odds for the logit model. Thus, for one unit change in the predictor variable, the odds for cases in a group less than or equal to a given size category of polyp are the proportional odds times larger.

${ }^{\mathrm{b}}$ Test for assumption of proportionality of odds across response categories, using a likelihood ratio test, showed that the assumptions of the ordinal logistic regression were met $(P=.190)$.

${ }^{c}$ Results reported are odds ratios for each variable in the model, adjusted for all other variables included in the model as shown here.

${ }^{\mathrm{d}}$ Family history was defined as a reported diagnosis of colon or rectal cancer in a mother, father, sibling, or child of the patient.

$* P<.001$

Thus, we found an additive, but not multiplicative effect of smoking on adenoma occurrence in the adjusted logistic regression models stratified by family history.

In addition to assessing presence or absence of an adenoma(s) as the outcome variable, we also assessed the association of smoking history with size of adenomas if present. Using an ordered logistic regression model we compared the likelihood of adenoma size $(<5 \mathrm{~mm}, 5-9 \mathrm{~mm}$, and $\geq 1 \mathrm{~cm}$ versus no adenoma) increasing in relation to smoking history. We found that, compared to nonsmokers, the highest-exposure smokers had $61 \%$ higher odds for the presence of an adenoma. The odds continued to increase proportionally for each successively larger adenoma size category $(\mathrm{OR}=1.61 ; 95 \%$ CI 1.34-1.93) indicating an association of larger adenoma size with greater smoking history in addition to simply adenoma occurrence (Table 5). Positive family history was also significantly associated with larger adenoma size (positive family history: OR $=1.69 ; 95 \%$ CI 1.37-2.08). Women had lower odds for larger adenomas $(\mathrm{OR}=0.54 ; 95 \%$ CI $0.46-0.63)$.

\section{Discussion}

We examined the proportion of patients in a screening colonoscopy population with multiple key risk factors for adenomatous polyps. This study extends the work of previous studies by characterizing the combined influence of the 
major risk factors: smoking, gender, and family history on adenoma occurrence.

As in other studies, we found a significantly greater proportion of adenomas among smokers. Pooled risk estimates from a recent meta-analysis of forty-two observational studies demonstrated a significant increase in likelihood of adenomas associated with smoking [9]. Many of these studies assessed the risk from smoking on adenoma characteristics, such as size, location, and number, and others assessed a host of single risk factors on adenoma occurrence. We report the combined effect of major risk factors on adenoma occurrence.

Although many studies have reported a lower risk of adenomas among women, and a higher risk with smoking, we additionally examined the joint effect of smoking and gender. The effect of smoking on adenoma occurrence seems to be different for men and women, with only males demonstrating an increased risk of adenoma with lower smoking exposure. Reasons for the differential effect of smoking on adenoma occurrence for women are not known but may be related to hormone replacement therapy, which was shown to decrease risk of adenomas [12, 13]. Calcium supplementation, which is higher among women, may also confer a protective effect to offset that of smoking $[14,15]$. Understanding the factors that may be protective for adenoma initiation in women should be a focus of investigation.

To our knowledge, we are the first to report that while smoking increases the risk of adenoma in screening patients with no family history of colorectal cancer or polyps, in the context of positive family history, the risk was similar for smokers and nonsmokers, particularly at the lower levels of smoking exposure. Anderson et al. characterized the effect of smoking in a screening population and demonstrated that smoking posed a greater risk than family history for prevalence of colorectal neoplasia [8]. Our results demonstrate that these risk factors in combination with each other do not exhibit a cumulative increase in risk.

A dominant strength of this study is the ability to analyze multiple risk factors. Further, we used a large colonoscopy registry, for which we have reliable measures of self report, and consistent pathology review. We were able to differentiate screening from surveillance colonoscopies, thereby more closely capturing incident adenomas.

We were somewhat limited in the number of cases for each risk factor, although this was likely to have limited only the number of factors upon which we could do stratified analyses. Greater numbers of cases would allow us to perform more detailed subgroup analyses, such as histological subtypes, adenoma size, colonic site, specific covariates, and more refined smoking exposure measures. Of particular interest would be to examine three- or fourway associations of smoking and other risk factors with adenomas.

In conclusion, we report an increased risk of adenomas and of larger adenomas with smoking, with the influence of smoking on adenoma occurrence being greater for men than women. Patients with no family history should be counseled about smoking as a significantly added risk factor for adenoma occurrence. For individuals at elevated risk due to family history, there does not appear to be additional, cumulative risk conferred by the combination of smoking and a family history. Further development and expansion of population-based colonoscopy registries would provide valuable data resources for more robust analyses, including detailed risk stratification, in order to optimize colorectal screening practices and reduce the incidence of colorectal neoplasms.

\section{Acknowledgment}

The National Cancer Institute (R21CA100553 and R01CA131141 supported this work.

\section{References}

[1] L. A. Cannon-Albright, D. T. Bishop, W. Samowitz, J. A. DiSario, R. Lee, and R. W. Burt, "Colonic polyps in an unselected population: prevalence, characteristics, and associations," American Journal of Gastroenterology, vol. 89, no. 6, pp. 827-831, 1994.

[2] E. Giovannucci, G. A. Colditz, and M. J. Stampfer et al., "A prospective study of cigarette smoking and risk of colorectal adenoma and colorectal cancer in U.S. women," Journal of the National Cancer Institute, vol. 86, no. 3, pp. 192-199, 1994.

[3] B.-T. Ji, J. L. Weissfeld, W.-H. Chow, W.-Y. Huang, R. E. Schoen, and R. B. Hayes, "Tobacco smoking and colorectal hyperplastic and adenomatous polyps," Cancer Epidemiology Biomarkers and Prevention, vol. 15, no. 5, pp. 897-901, 2006.

[4] J. Kearney, E. Giovannucci, and E. B. Rimm et al., "Diet, alcohol, and smoking and the occurrence of hyperplastic polyps of the colon and rectum (United States)," Cancer Causes and Control, vol. 6, no. 1, pp. 45-56, 1995.

[5] D. A. Lieberman, S. Prindiville, D. G. Weiss, and W. Willett, "Risk factors for advanced colonic neoplasia and hyperplastic polyps in asymptomatic individuals," Journal of the American Medical Association, vol. 290, no. 22, pp. 2959-2967, 2003.

[6] E. D. Paskett, K. W. Reeves, and B. Pineau et al., "The association between cigarette smoking and colorectal polyp recurrence (United States)," Cancer Causes and Control, vol. 16, no. 9, pp. 1021-1033, 2005.

[7] M. B. Terry, A. I. Neugut, and R. M. Bostick et al., "Risk factors for advanced colorectal adenomas: a pooled analysis," Cancer Epidemiology Biomarkers and Prevention, vol. 11, no. 7, pp. 622-629, 2002.

[8] J. C. Anderson, R. Attam, and Z. Alpern et al., "Prevalence of colorectal neoplasia in smokers," American Journal of Gastroenterology, vol. 98, no. 12, pp. 2777-2783, 2003.

[9] E. Botteri, S. Iodice, S. Raimondi, P. Maisonneuve, and A. B. Lowenfels, "Cigarette smoking and adenomatous polyps: a meta-analysis," Gastroenterology, vol. 134, no. 2, pp. 388-e3, 2008.

[10] L. M. Morimoto, P. A. Newcomb, C. M. Ulrich, R. M. Bostick, C. J. Lais, and J. D. Potter, "Risk factors for hyperplastic and adenomatous polyps: evidence for malignant potential?" Cancer Epidemiology Biomarkers and Prevention, vol. 11, no. 10, part 1, pp. 1012-1018, 2002.

[11] P. A. Carney, M. E. Goodrich, L. F. Butterly, and A. J. Dietrich, "The design and development of a population-based colonoscopy registry," Journal of Registry Management, vol. 33, no. 4, pp. 91-99, 2006. 
[12] M.-J. Chen, M. P. Longnecker, H. Morgenstern, E. R. Lee, H. D. Frankl, and R. W. Haile, "Recent use of hormone replacement therapy and the prevalence of colorectal adenomas," Cancer Epidemiology Biomarkers and Prevention, vol. 7, no. 3, pp. 227230, 1998

[13] M. P. Purdue, P. J. Mink, P. Hartge, W.-Y. Huang, S. Buys, and R. B. Hayes, "Hormone replacement therapy, reproductive history, and colorectal adenomas: data from the Prostate, Lung, Colorectal and Ovarian (PLCO) Cancer Screening Trial (United States)," Cancer Causes and Control, vol. 16, no. 8, pp. 965-973, 2005.

[14] M. V. Grau, J. A. Baron, and R. S. Sandler et al., "Vitamin D, calcium supplementation, and colorectal adenomas: results of a randomized trial," Journal of the National Cancer Institute, vol. 95, no. 23, pp. 1765-1771, 2003.

[15] K. Wu, W. C. Willett, C. S. Fuchs, G. A. Colditz, and E. L. Giovannucci, "Calcium intake and risk of colon cancer in women and men," Journal of the National Cancer Institute, vol. 94, no. 6, pp. 437-446, 2002. 


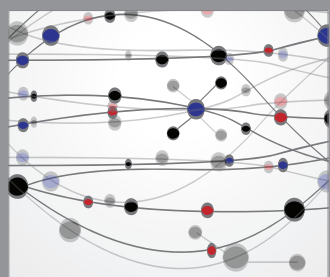

The Scientific World Journal
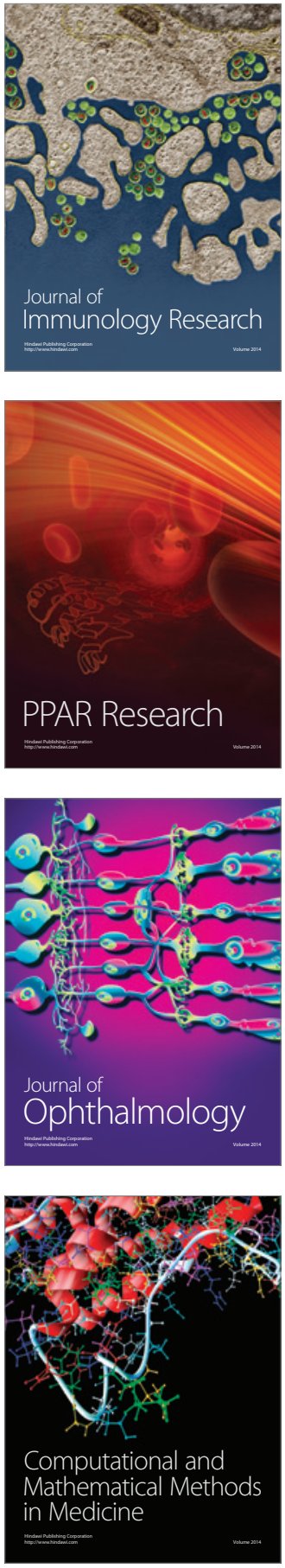

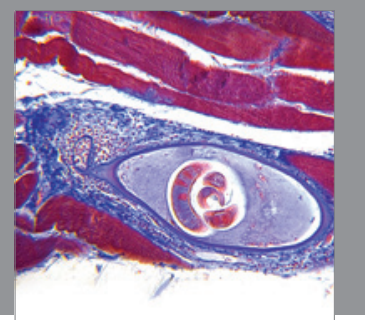

Gastroenterology

Research and Practice
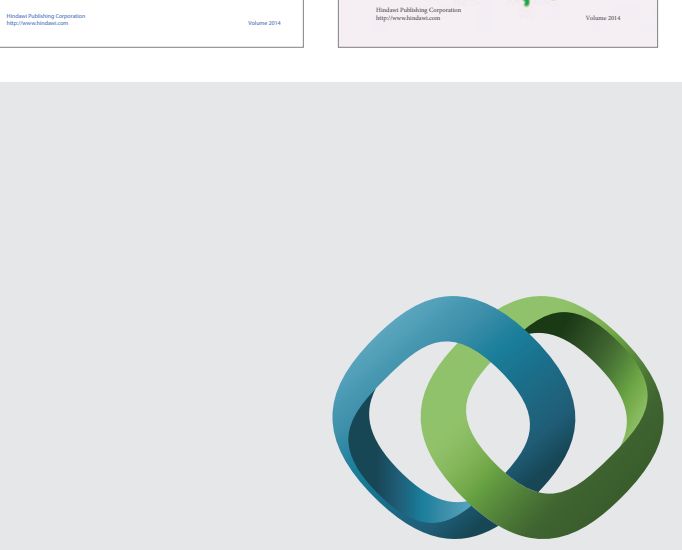

\section{Hindawi}

Submit your manuscripts at

http://www.hindawi.com
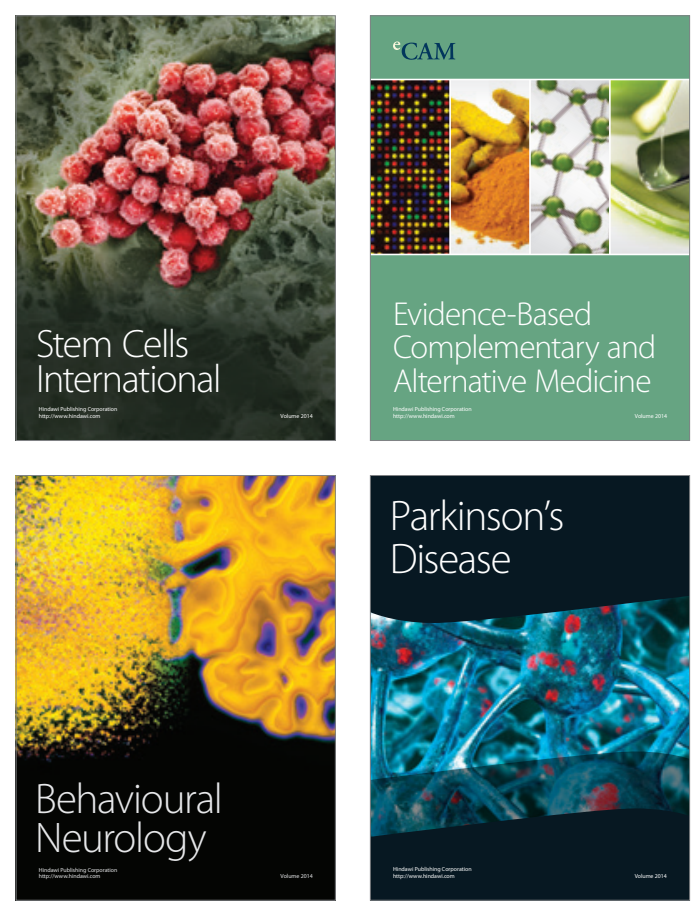

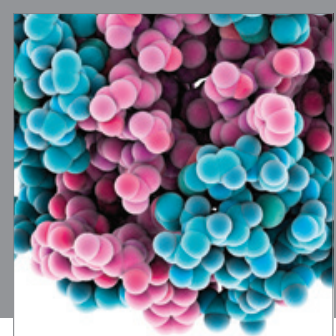

Journal of
Diabetes Research

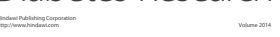

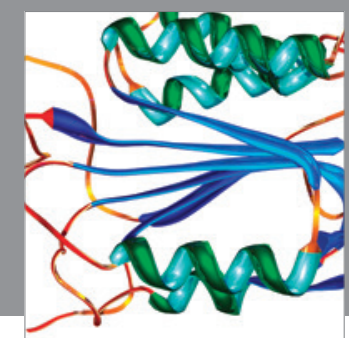

Disease Markers
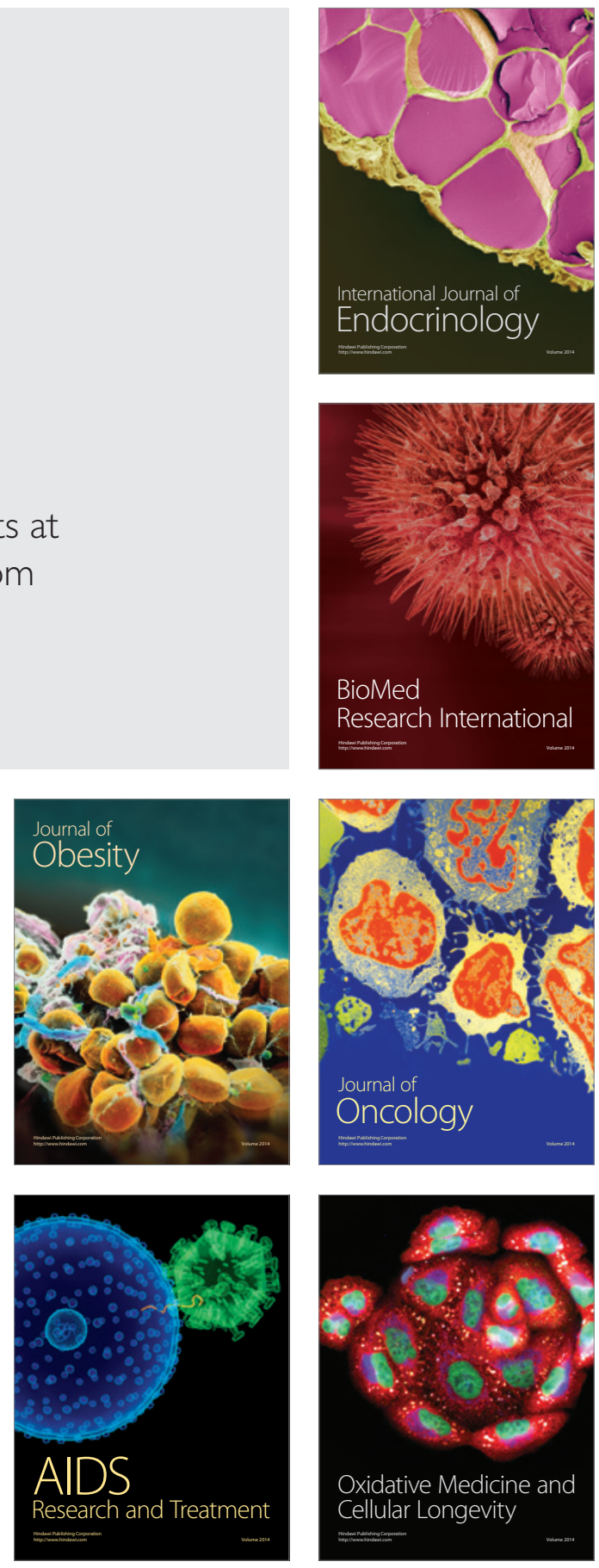